\title{
Age-related Gender Differences in Motor and Inhibitory Learning and Consolidation
}

\author{
T. Pereira ${ }^{1,2}$, A. Castro-Caldas ${ }^{2}$ and A.M. Abreu ${ }^{3,{ }^{*}}$ \\ ${ }^{1}$ Institute of Health Sciences, Portuguese Catholic University, Lisbon, Portugal \\ ${ }^{2}$ Centro de Investigação Interdisciplinar em Saúde, Universidade Católica Portuguesa, Lisbon, Portugal \\ ${ }^{3}$ Universidade Europeia, Laureate International Universities, Lisbon, Portugal
}

\begin{abstract}
Aim: The understanding of the neural correlates of motor learning and consolidation has seen significant progress in recent years. Such advances have afforded the development of better training plans and the potentiation of motor skill learning in sports, in neurological recovery or simply in everyday life. However, the variations in motor learning and consolidation across different ages are still not well understood. In order to investigate this, we assessed performance in two different tasks (Finger Tapping Sequence and Go/No-Go tasks) in four different Age groups (Children; Young Adults; Mature Adults, and Seniors).

Materials and Methods: The two tasks were executed across three different time periods (T0, T1 and T2), during which performance was measured: Day 1. Baseline (T0) and Performance After Training - i.e. Learning (T1) and; Day 2. Consolidation Performance - 24 hours post-T1 without any additional training (T2).

Results: We show that the group of Seniors did not enhance performance 24 hours post-training in the Finger Tapping Sequence task, while all the other Age groups did. There were no differences in performance in Children, but age and sex interacted to enhance performance. This complex mechanism was shown to be task-specific. Moreover, none of the Age groups enhanced performance in T2 in the Go/No-Go Task, but we found a female advantage after practice in Mature Adults and Seniors.

Conclusions: The influence of both Age and Sex in task performance and consolidation is to be taken into consideration in order to ameliorate training and potentiate individual capacities while delaying age-related impairments.
\end{abstract}

Keywords: Motor learning, Motor consolidation, Development, Aging.

\section{INTRODUCTION}

Learning a new set of motor skills as well as relearning previously acquired ones is fundamental not only for everyday life activities, but also constitutes an extremely important means to improve rehabilitation programmes following brain injuries that affect motor performance [1]. Amelioration in motor performance can be achieved via online gains reported during training sessions and also by means of offline gains without additional training [2]. These offline gains can be modulated by the structure of practice [3], and are of paramount importance, as they represent additional motor performance gains after the end of a training session without additional training. In a previous study with young adults, we showed that different motor tasks could have different off-line gains [4]. Here, we aim to investigate how these off-line gains are modulated across the lifespan.

A considerable amount of research has been dedicated to the psychophysical consequences of aging, focusing on how to avoid impairments in motor

"Address correspondence to this author at Universidade Europeia, Laureate International Universities, Lisbon, Portugal; Tel: (00351) 210309900;

Fax: (00351)210309917; E-mail: ana.abreu@europeia.pt learning and re-learning, essential for our daily activities, and on the mechanisms and procedures that allow the potentiation of motor and cognitive memory acquisition and recall across different ages [e.g., 5-7]. It has been well established that cognitive-motor activities facilitate neuro-protection [8]. However, the specific importance of motor activity and mental exercise for the brain across different Age groups is still unclear. Specifically, we know that there can be an increase in the brain's white matter by training working memory in older adults [9]. However, these findings report to training in cognitive tasks. But what consequences are expected when training occurs in motor tasks? Such a query was addressed in a study where older adults are shown to be able to shift between implicit and explicit learning when a new motor sequence is being learned [10]. Despite this ability to alternate between learning types, most studies have focused more on degeneration and impairment in brain functions with age, and less on learning capabilities [e.g., 11-15]. Accordingly, most studies have investigated physical exercise and motor behaviour as ways to enhance otherwise deteriorated performance in older subjects [e.g., 16, 17]. One such example relates to the benefits brought by physical 
exercise that can be mediated by the enhancement of Brain-Derived Neurotrophic Factor - BDNF [18] that can positively interfere with some neurodegenerative diseases, such as Alzheimers' Disease [19]. Moreover, physical activity has been shown to enhance time response in older and in middle-aged people, and ameliorate the planning/execution of a response as well as the executive functions mediated by the prefrontal cortex [20]. Learning and consolidating a motor sequence task seems to activate cortical and sub-cortical structures such as the basal ganglia, cerebellum, supplementary motor, primary motor and premotor areas [21-24]. Furthermore, some authors found the hippocampus to be implicated, not just on the learning phases, but also in the consolidation phases of a motor memory $[25,26]$. It is possible that some neurodegenerative diseases that typically occur in older ages, in which the functioning of the hippocampus is compromised [e.g., 27], might consequently affect the process of motor memory consolidation. Two other structures involved in the initial phase of motor sequence learning are the frontal cortex and the striatum. Accordingly, task-dependent deficits may be attributed to the age-associated degeneration in cortico-striatal networks [28, 29]. However, recent neuroimaging studies shed new light on the aging brains' function and it seems that a better way to describe connectivity in the brain's networks in the mature brain is not by means of describing its deficits, but by understanding its changes in functionality that may decline, maintain or even improve [30]. Specifically, age-related changes in connectivity are consistent with increased emotion regulation and decreased cognitive functions [31]. Moreover, preservation of cognitive functions in older adults has been associated to compensatory mechanisms associated to neuroanatomical and functional changes leading to an overall increase in, albeit less efficient, functional connectivity [32]. Crucially, and in what pertains to our study, aging may alter the connectivity of brain networks underlying motor learning by increasing the bilateral-frontal and fronto-parietal connectivity [33].

Although it has been well established that motor performance tends to decline in older ages [34], and that healthy older subjects experience significant declines in motor skill acquisition when compared to younger subjects [35], it remains unclear how off-line enhancements are modulated with motor memory consolidation across age. As such, contrasting data arise from a study by Smith and collaborators [36] showing an age resistant component of motor memories, compared to declines in motor learning and performance with age. Very little data is available to add on to this discussion, Dorfberger and collaborators [37] have shown no differences when learning or retaining new motor memories, between children and adolescents. However, children were less susceptible to interference when compared to adolescents: i.e., newer motor memory experiences affect the consolidation process of previously learned motor memories in young adults, but not in children. Nevertheless, these authors did not compare these younger groups with adults or seniors. According to these and the aforementioned data, there are several brain and behavioural changes that occur across the lifespan that might compromise the ability to learn and consolidate new motor memories. This can have a tremendous impact in the aging persons' everyday life, as lifelong motor learning should help increase and maintain motor independence.

It thus seems paramount to investigate motor performance and consolidation across the lifespan. Specifically, the capacity to learn and consolidate a novel motor sequence or simply to perform an action and/or to inhibit that same action on cue, are important requisites in everyday life activities. Here, we will investigate these exact capabilities. Finally, it is important to note that previous research has already demonstrated the existence of sex differences in motor task learning, but not performance, given by a male advantage, enhanced during adolescence compared to younger groups [38]. However, and to the best of our knowledge, the analysis of sex differences throughout the lifespan, from infancy to old age, has not been described and is mostly ignored due to the structure of the protocols. Here, we attempt to discriminate the influence of gender in age-related motor learning and consolidation and we discuss our results in light of the latest neuroscientific available data pertaining to functional and architectural changes across the lifespan.

\section{METHODS}

\section{Participants}

One hundred and twenty eight neurotypical subjects (64 Male and 64 Female) participated in this study and were divided into four Age groups: 32 (16 Female) Children (aged 8-9 years old; $M=8.75 ; S D=0.44$ years); 32 (16 Female) Young Adults (aged 20-25 years old; $M=21.88 ; S D=1.57$ years); 32 (16 Female) 
Mature Adults (aged 40-45 years old; $M=42.58$; $S D=$ 1.98 years) and 32 (16 female) Seniors (aged 65-70 years old; $M=67.29$; $S D=2.05$ years). The participants were recruited from primary schools, universities, local workplaces and nursing homes (from the Algarve area, Portugal), respectively. All participants were right handed and had no outstanding medical condition that might impair fine motor performance.

\section{Procedures}

Participants and the participants' parents or guardians (in the case of minors) gave their written informed consent prior to participating in the experimental tasks and received information concerning the experimental procedures. This study was conducted in accordance with the tenets of the Declaration of Helsinki (2008). The participants' performance was assessed in two different tasks (Finger Tapping Sequence and Go/No-Go Tasks) in order to investigate motor learning, and consolidation across different Age groups. The assessment was conducted in accordance with the procedures previously described by Pereira and collaborators [4]. Each task was first performed in a Training Session (T0 to T1). During this session, Baseline Performance - T0 (first time the subject executed the task) and Performance after training - T1 (average of the last 3 trials) - were assessed. Subsequently, all subjects were re-tested on the same tasks, after a 24-hour delay without additional training (Consolidation Session -T2). The 24-hour period without additional training, allowed for consolidation, as described in previous designs [e.g., 39]. Each participant was randomly assigned to start out with either the Finger Tapping Sequence or the Go/No-Go Task. Participants were tested in a silent and dimly lit room with the fewest distractions as possible. Participants were instructed to have a good night of sleep (7 to 9 hours) between T1 and T2 (24 hour-period). All subjects met this inclusion criterion.

\section{Apparatus}

Both tasks were presented on a computer screen and participants were seated at a distance of $\pm 60 \mathrm{~cm}$ from the computer screen. For the Finger Tapping Sequence, participants were instructed to tap a five number sequence on the computer keyboard (task described below). The Go/No-Go task, on the other hand, was developed using Super Lab 4.5 and was also presented on a computer screen. Here, the aim was to either respond motorically or inhibit the motor response to the stimuli presented on the computer screen as described below. In both tasks, the participants were instructed to tap the sequence, press a button or inhibit the motor response as quickly and accurately as possible.

\section{TASKS}

\section{Finger Tapping Sequence}

As reported in previous studies [e.g., 40, 41], learning a novel motor task, such as the Finger Tapping Sequence task, should progress through a series of unique memory stages. Specifically, performance should initially improve during training and continue to improve even without additional training after a 24-hour period. Here we intended to verify how such performance might be modulated across different Age groups and sexes.

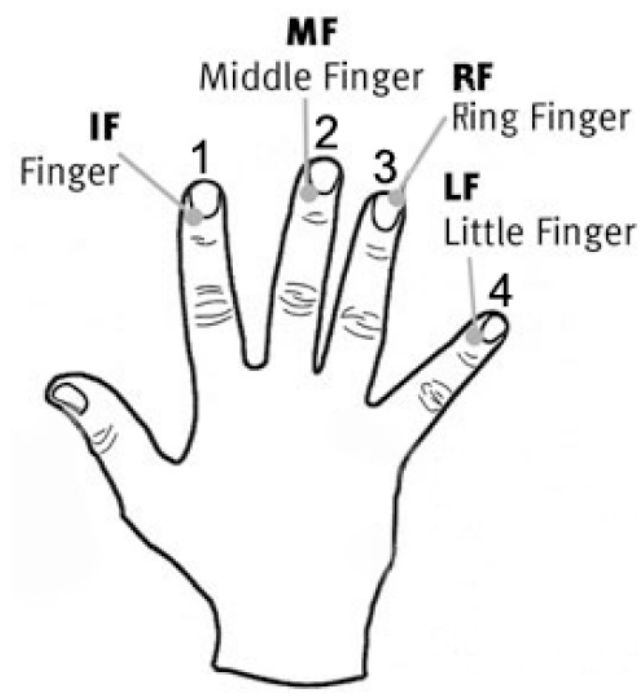

Figure 1: Digit-to-finger correspondence for the Finger Tapping Sequence task (Sequence: 4_1_3_2_4).

The participants were required to learn a Finger Tapping Sequence (4_1_3_2_4) by using a computer keyboard. The finger sequence corresponded to computer keys as follows: digit 1 - Index finger; digit 2 - Middle finger; digit 3 - Ring finger; digit 4 - Little finger (see Figure 1). The participants were requested to repeat the sequence as quickly and as accurately as possible for 30 seconds. Each 30 -second trial was initiated and terminated by an auditory signal cue. The participants were instructed to tap the movement sequence continuously until hearing the stop signal, and to continue without pause, even if committing any error, as quickly and accurately as possible. Performance was given by the number of correctly typed sequences. The training session consisted of twelve 30 -second trials with 30 -second rest periods in 
between trials lasting $\pm 12 \mathrm{~min}$ in total [42]. Baseline Performance (T0) consisted on the first 30-second trial, whereas Performance After Training (T1) was given by the mean of the last three trials. The Consolidation Session (T2) consisted of three 30-second trials with 30 -second rest periods between trials, executed 24 hours post-T1, without additional training. T2 performance consisted on the mean of all three 30second trials executed during the consolidation session. All sequences were recorded on the computer throughout the completion of the trials.

\section{Go/No-Go Task}

Understanding the control of motor inhibition is extremely important, as it constitutes a significant part of everyday life. Inhibitory control is an executive function, i.e. a higher cognitive function involved in the executive control of behaviour that has been linked to motor coordination [43]. Certain fast inhibitory actions are not processed at a conscious level and are in close relation with the response reaction time to a certain stimulus. Hence, a better understanding of inhibitory performance might help in developing new motor learning strategies. In order to tap this issue, we assessed performance in a Go/No-Go task across different Age groups and sexes.

The participants were requested to answer to arrow stimuli presented on a computer screen as fast and accurately as possible. The stimuli consisted of four arrows (green, right or left and red, right or left) that were randomly presented (60 arrow presentations in total, per block). Each arrow stimulus remained

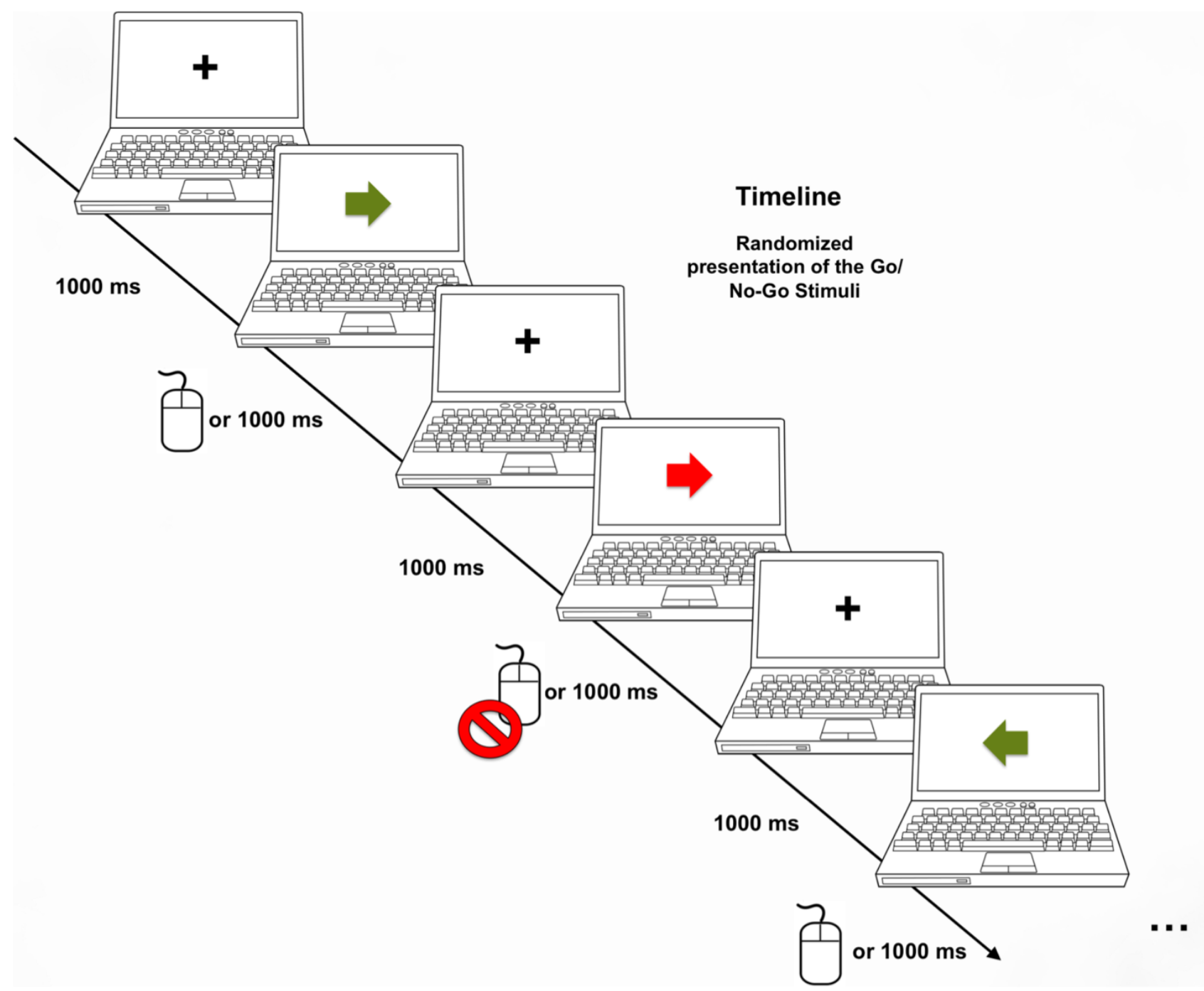

Figure 2: Go/No-Go task. Stimuli timeline. Each of the four arrows (red left and right and green left and right) was presented randomly for 1000 milliseconds interspersed with fixation crosses. Participants were instructed to press the right mouse button in response to the right green arrow; the left mouse button in response to the left green arrow and to refrain from pressing any button in the presence of any of the two red arrows. 
onscreen for $1000 \mathrm{~ms}$, after which the participant could no longer answer. The participants were instructed to press the right mouse button as a response to a green arrow to the right, to press left mouse button as a response to a green arrow to the left and to refrain from pressing any button when any of the red arrows (left or right) were presented (see Figure 2). The participants were also instructed to answer as quickly and accurately as possible. When a mouse button was pressed the fixation cross would immediately appear. If no answer took place, the fixation cross would appear 1000 ms later. In either case, a fixation cross trial would always intersperse the arrow trials. The experiment included twelve 60-trial blocks (one trial $=$ one arrow) with 30 second rest periods between blocks, lasting $\pm 30 \mathrm{~min}$ in total. This procedure was used to maintain a similar protocol and rest periods as in the Finger Tapping Sequence task. The arrows were randomly presented and were $50 \%$ green and $50 \%$ red, in order to avoid motor learning. Although, in the Finger Tapping Sequence task, performance was only given by accuracy, here, performance was measured by means of two dependent variables: a) Speed - given by the reaction time to respond to the green arrows (measure of speed) and; b) Accuracy - given by [1 the number of errors made (measure of accuracy)]. Errors were computed when one either pressed the right button when a left arrow was presented, or pressed the left button in view of a right arrow, or simply pressed any button in the presence of a red arrow. Both accuracy and speed were recorded by the Super Lab software used to develop the stimuli.

In order to integrate both speed and accuracy in one overall index of performance [e.g. 44, 45], we computed the Inverse Efficiency Score (IES) by dividing, for each Time condition (T0, T1, \& T2) and in each subject, the mean correct reaction time by the percentage of correct responses. The IES was introduced by Townsend and Ashby [46] to control for speed-accuracy trade-off effects (i.e. when movement speed increases, movement accuracy decreases [47], as it combines accuracy and reaction times in a single measure. Worse overall performance is given by higher scores.

In conformance with the Finger Tapping Sequence task, we assessed IES at TO that consisted on the ratio between the mean RT and the proportion of correct responses obtained during the first block constituted by 60 trials; IES_T1, on the other hand, consisted on the ratio between the mean $\mathrm{RT}$ and the proportion of correct responses obtained during the last three 60-trial blocks of the training session. Finally, IES_T2 consisted on the ratio between the mean RT and the proportion of correct responses obtained from the average of the three 60 -trial blocks executed 24 hours after training.

\section{RESULTS}

In order to investigate motor learning and consolidation performance, we considered performance measurements across three different time periods for the two tasks, in the four Age groups. Here, we aimed to investigate performance given by accuracy in the Finger Tapping Sequence task, and by accuracy and speed integration (i.e. the speed accuracy trade-off) in the Go/No-Go task across the different time periods (Learning and Consolidation) and across different Age groups.

The data were entered into separate $4 \times 2 \times 3$ Mixed Repeated Measures Factorial ANOVAs (Group $x$ Sex $x$ Time), with group type (Children $x$ Young Adults $x$ Mature Adults $x$ Seniors) and Sex (Male $x$ Female) as between-subjects covariates and time of measurement as within-subjects factors ( $\mathrm{T} 0 \times \mathrm{T} 1 \times \mathrm{T} 2$ ), for each task. Bonferroni corrected Post hoc multiple comparisons were performed.

\section{Finger Tapping Sequence Task}

Concerning the Finger Tapping Sequence task, we computed a Repeated Measures ANOVA, as stated above, and found a significant main effect of Time ( $F$ $(2,240)=492.170, p=0.000)$. Bonferroni adjusted pairwise comparisons indicate that there were performance gains from $\mathrm{TO}(\mathrm{M}=4.133$; $\mathrm{SE}=0.203)$ to $\mathrm{T} 1(\mathrm{M}=8.070 ; \mathrm{SE}=0.201)$ to $\mathrm{T} 2(\mathrm{M}=10.039$; $\mathrm{SE}=$ 0.252 ) (all $P=0.000$ ). Globally, the participants enhanced their performance from baseline assessment, to assessment after training to the 24 hours post-training assessment (i.e. off-line gains that enhance motor performance without additional training after a 24 hour interval). We also found a significant main effect of Age-group $(F(3,120)=194.126, p=$ 0.000). Bonferroni adjusted pairwise comparisons indicate that Young Adults ( $M=13.563$; $S E=0.378$ ) outperform Mature Adults $(M=7.781$; $S E=0.378)$, whom in turn, outperform Children $(\mathrm{M}=5.417$; $\mathrm{SE}=$ $0.378)$, all of which outperform Seniors ( $M=2.896$; SE $=0.378$ ) (all $P=0.000)$. On the other hand, we did not find a significant main effect of $\operatorname{Sex}(F(1,120)=1.502$, $p=0.223)$, which is to say that gender per se did not modulate performance in the Finger Tapping Sequence task. 
We did, however, find two-way interaction effects between Time and Age-Group $(F(6,240)=37.204, \mathrm{p}=$ $0.000)$ and between Time and $\operatorname{Sex}(F(2,240)=22.179$, $\mathrm{p}=0.000)$. Finally, we report a three-way interaction effect between Time, Age-Group and Sex $(F(3,120)=$ 2.707, $\mathrm{p}=0.015)$. Bonferroni adjusted pairwise comparisons show that the off-line enhancement of performance (Consolidation), without additional training, did not occur in Mature Adult Males $(p>0.05)$ and in Female Seniors $(p>0.05)$. Moreover, not only did Male Seniors not benefit from 24-hour post-training off-line gains, they also did not benefit from training in the first place (i.e. in the Male Senior group, T0 does not differ from T1 or T2 and T1 and T2 do not differ between them, all $P>0.05$ ). All other comparisons were highly significant, showing across-group gains from $\mathrm{T} 0$ to $\mathrm{T} 1$ to $\mathrm{T} 2$ (all $\mathrm{P}=0.000$ ). Furthermore, Bonferroni adjusted multiple comparisons show differences between Sex groups, according to Time and Age groups. In T0, only Young Male ( $M=10.875$; $\mathrm{SE}=0.511)$ and Young Female Adults $(M=5.375$; SE $=0.511)$ differ in their performance $(p=0.000)$, as Males outperform Females; In T1, both Young Male (M
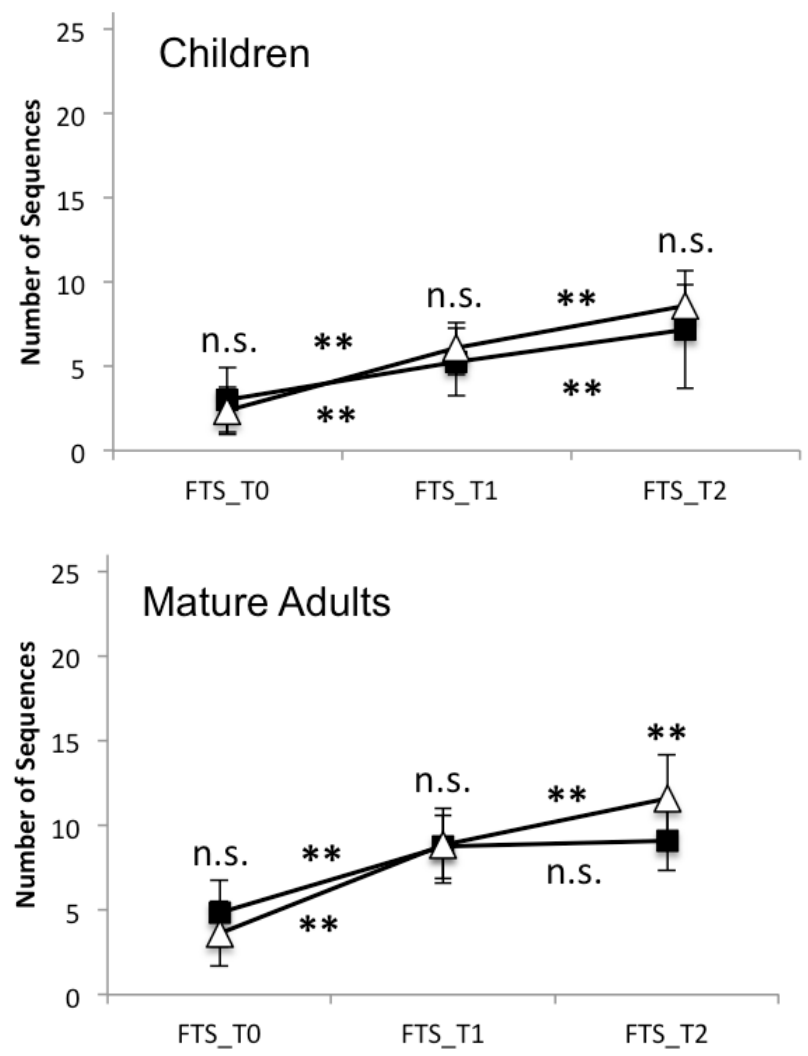

$=15.563 ; \mathrm{SE}=0.535)$ and Young Female Adults $(\mathrm{M}=$ 13.125; $S E=0.535)(p=0.002)$, and Male $(M=2.438$; $\mathrm{SE}=0.535)$ and Female Seniors $(M=4.563$; $\mathrm{SE}=$ $0.535)(p=0.006)$ differ in their performance. However, while Young Male Adults outperform Females, we find the opposite pattern in Seniors, with Females outperforming Males; Finally, in T2, only Children present similar performances between sexes $(p>$ $0.05)$, while Young Male $(M=20.375$; $S E=0.624)$ and Young Female Adults $(M=16.063 ; S E=0.624)(p=$ $0.00)$, Mature Male $(M=9.125 ; S E=0.624)$ and Mature Female Adults $(\mathrm{M}=11.563 ; \mathrm{SE}=0.624)(\mathrm{p}=$ $0.00)$, and Male $(M=2.438 ; S E=0.624)$ and Female Seniors $(M=4.938$; $S E=0.624)(p=0.00)$, show differences in performance, with Males outperforming Females as Young Adults and losing this advantage as Mature Adults and Seniors (see Figure 3).

\section{Go/No-Go Task}

The IESs were entered into a Repeated Measures ANOVA using the same procedure as in the Finger Tapping Sequence task analysis. As with the previous
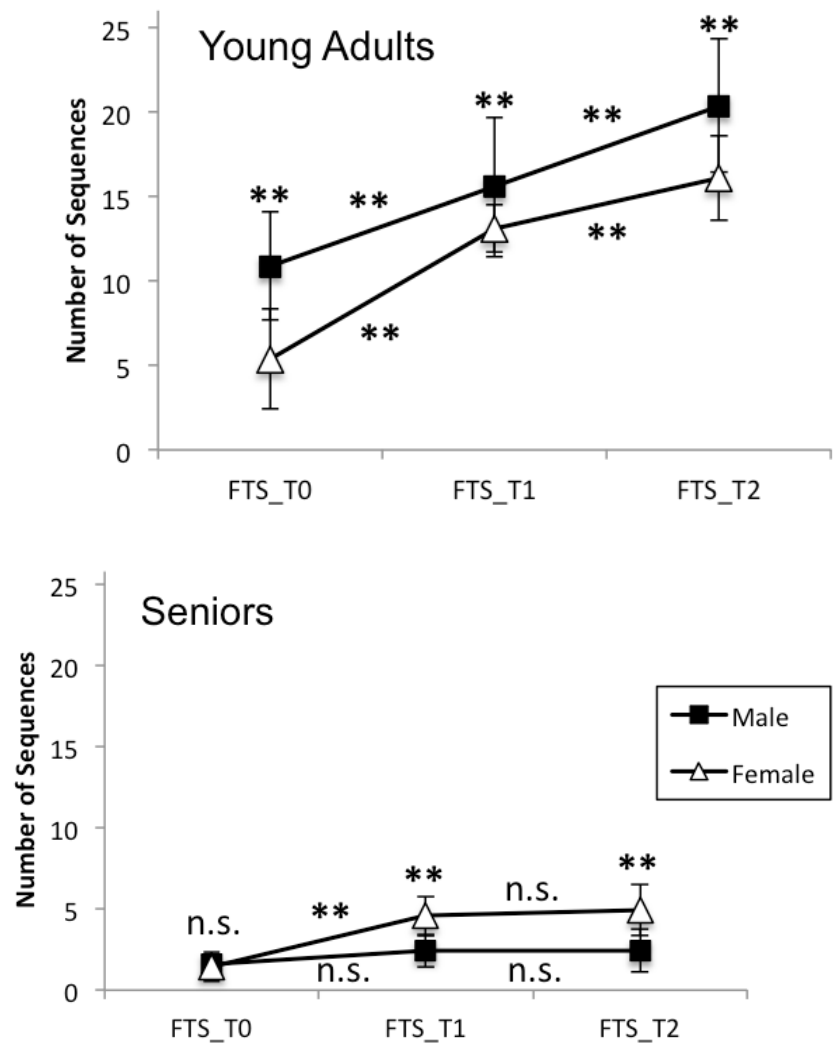

Figure 3: Across-session performance gains for males and females of the four age groups in the Finger Tapping Sequence task. Performance accounts for mean number of correct sequences. FTS_T0 consists on the mean number of sequences in the first 30-sec trial; FTS_T1 consists on the mean number of sequences in the last three trials of the training session; FTS_T2 consists on the mean number of sequences in the three $30-\mathrm{sec}$ trial executed 24 hours after training. Bars represent standard deviation of the mean. ${ }^{* *}$ indicate significant comparisons $(p<0.01)$ 
task, we found a significant main effect of Time $(F(2,240)=33.159, \mathrm{p}=0.000)$. Bonferroni adjusted pairwise comparisons indicate that there were performance gains from $\mathrm{TO}(\mathrm{M}=926.822$; $\mathrm{SE}=$ $27.151)$ to $\mathrm{T} 1(\mathrm{M}=786.142 ; \mathrm{SE}=16.155)$ to $\mathrm{T} 2(\mathrm{M}=$ 742.726; SE $=16.488$ ) (all $P<0.05$, higher scores indicate worse performances). As before, the participants enhanced their performance from baseline assessment to assessment after training to the 24 hours post-training assessment. Although subtle, the off-line gains without additional training after a 24 hourinterval were significant. Once again, we found a significant main effect of Age-Group $(F(3,120)=$ 123.297, $\mathrm{p}=0.000$ ). Bonferroni adjusted pairwise comparisons indicate that Young Adults ( $M=457.098$; $S E=30.798)$ outperform Mature Adults $(M=812.085$; SE $=30.798)$ and the Children's group $(M=726.440$; SE $=30.798)$ (both $P=0.000)$, although Mature Adults and Children do not present any differences in performance ( $p=0.309)$, all groups outperform Seniors $(\mathrm{M}=1278.632 ; \mathrm{SE}=30.798)$ (all $P=0.000)$. In striking contrast with the Finger Tapping Sequence task, the Go/No-Go task does present a significant main effect of
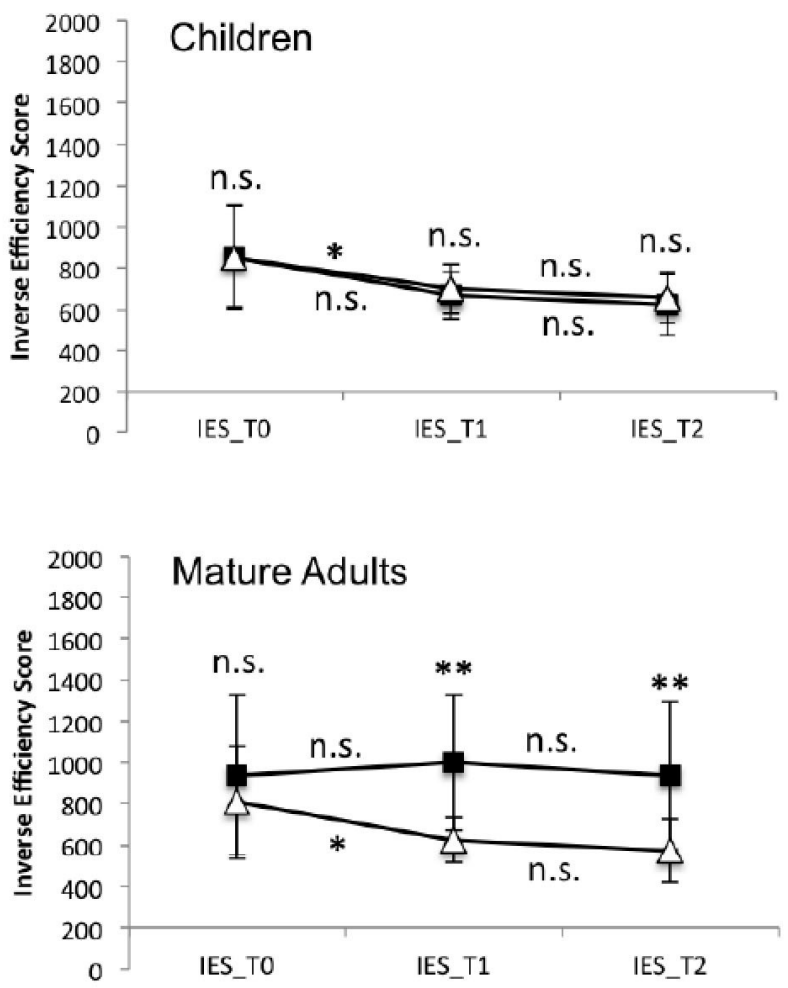

Sex $(F(1,120)=29.025, \mathrm{p}=0.000)$, which is to say that gender per se does modulate performance in the Go/No-Go task, whereby Females $(\mathrm{M}=735.601$; $\mathrm{SE}=$ 21.778) outperform Males $(M=901.526$; $S E=21.778)$, $\mathrm{p}=0.000$.

Again, we found an interaction effect between Time and $\operatorname{Sex}(F(2,240)=7.686, \mathrm{p}=0.001)$, however, no interaction effect between Time and Age-Group was found $(F(6,240)=2.017, \mathrm{p}=0.064)$, this is to say that performance across time is not differently modulated by the different Age Groups. Finally, we report a three-way interaction effect between Time, Age-Group and Sex ( $F$ $(6,240)=6.371, p=0.000)$. Bonferroni adjusted pairwise comparisons show that the off-line enhancement of performance (Consolidation), without additional training, did not occur in any of the Age groups ( $p>0.05$ ) and improvement from T0 to T1 in overall IES occurred only in Children $(p=0.002)$ and Seniors $(p=0.002)$. However, when considering the sexes separately, we find that differences between T0 and $\mathrm{T} 1$ are supported only by Male Children ( $p=$ 0.020). Moreover, we uncover a significant enhancement in Mature Female Adults, from T0 to T1
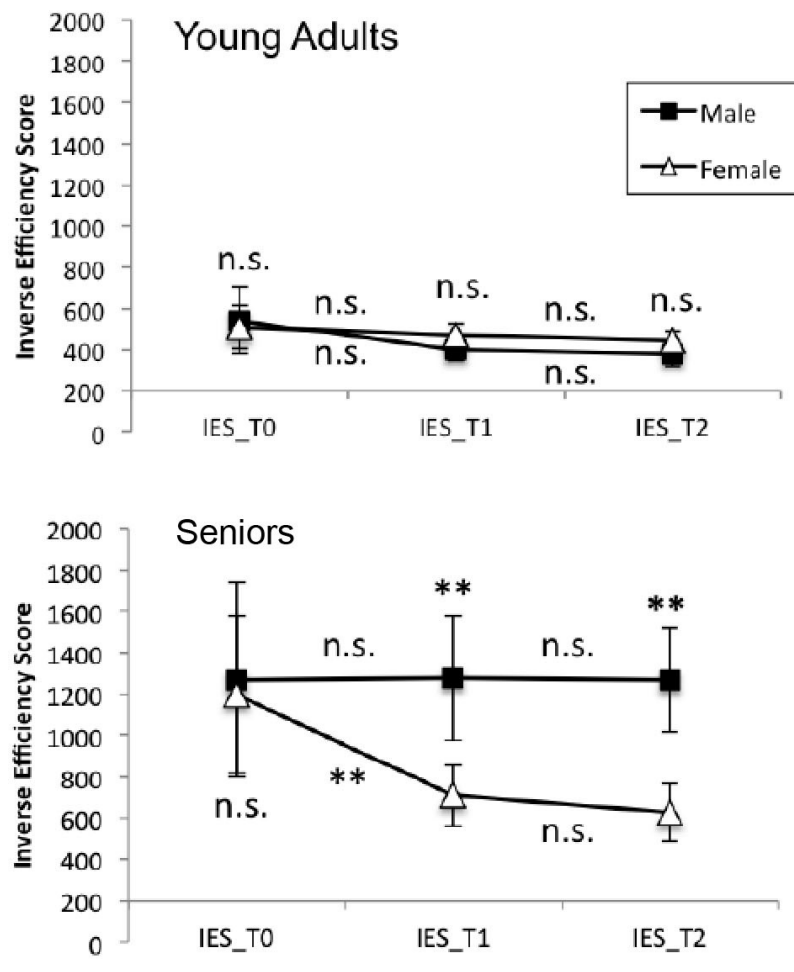

Figure 4: Across-session performance gains for males and females of the four age groups in the Go/No-Go task. Performance for IES_T0 consists on the ratio between the mean RT and the proportion of correct responses obtained during the first block constituted by 60 trials; IES_T1 consists on the ratio between the mean RT and the proportion of correct responses obtained during the three last 60-trial blocks of the training session; IES_T2 consists on the ratio between the mean RT and the proportion of correct responses obtained from the average of the three 60-trial blocks executed 24 hours after training. Bars represent standard deviation of the mean. *Indicates significant comparisons $(p<0.05)$ ** indicate significant comparisons $(p<$ $0.01)$. 
$(p=0.024)$ and verify that the amelioration in performance, from T0 to T1, in Seniors was also due to the Female participants $(p=0.000)$. So it seems that training benefits Male Children and Mature and Senior Female participants, while off-line gains without training, i.e., consolidation, does not occur in any Sex or Age-group. Importantly, across time, Children's performance $(\mathrm{M}=855.184 ; \mathrm{SE}=54.301)$ equals that of Mature Adults $(\mathrm{M}=872.447$; $\mathrm{SE}=54.301)$ in T0; $(\mathrm{p}=$ 1.000 ), but is worse than that of Young Adults ( $M=$ 525.383; SE $=54.301$ ) and better than that of Seniors $(\mathrm{M}=1454.274$; $\mathrm{SE}=54.301)$; (both $\mathrm{P}=0.000)$. After Training, in $\mathrm{T} 1$, on the other hand, differences in performance between Children $(\mathrm{M}=684.822$; $\mathrm{SE}=$ 32.311) and Mature Adults $(M=810.594 ;$ SE $=32.311)$ arise, as Children outperform Mature Adults $(\mathrm{p}=$ 0.041). Finally, in $T 2$, these differences are lost as Children ( $\mathrm{M}=639.313$; $\mathrm{SE}=32.975)$ and Mature Adults $(\mathrm{M}=753.214$; $\mathrm{SE}=32.311)$, both show similar performances $(p=0.096)$. Conversely, all other Age groups perform differently across time-points as discussed above (see Figure 4).

\section{DISCUSSION}

One of our main findings points to a lack of consolidation effect in Seniors in both tasks. As in previous investigations, older adults are the only group where no positive effects of consolidation can be imprinted on performance. These results are in accordance with previous studies where older adults do not show consolidation benefits in motor sequence tasks [48-50]. Although our results show the existence of offline gains in three of the four Age groups (Children, Young Adults and Mature Adults), these gains are only associated to the finger Tapping Sequence Task. It is possible that the neural requirements involved in motor memory establishment (implicated in the consolidation of the Finger Tapping Sequence task), might not translate equally in a task that predominantly involves online decision-making (as required by the Go/No-Go task). This is particularly curious given that Verbruggen and Logan [51] have shown that stimulus-stop associations can indeed be trained in Go/No-Go tasks. It thus seems that when it comes to consolidation, both task and/or Age-group might have an effect. These differences can be associated with the age degeneration that occurs in cortico-striatal networks [28, 29] that might be differently implicated in motor memory and inhibition control tasks. This is consistent with previous studies showing that different executive functions result from the interplay of different cortical systems [52].
Concurrently, overall performance can be differently modulated by sexes according to Age-group, as shown predominantly in Young and Mature Adults and Seniors, but not in Children. Previous studies had already shown that motor performance generally improves from childhood to young adulthood and from there, decreases well into old age [53]. Other studies have consistently shown a growing male advantage in motor performance from childhood to adolescence [e.g., 38]. However, and to the best of our knowledge, none had investigated the complex interactions between sexes and age (from childhood to old age), as we have tapped here. According to previous studies $[40,41]$ there is a great effect of consolidation on performance that leads to enhancements of around 20 to $30 \%$ in motor sequence performance. Our results reveal that this performance enhancement without additional training only occurs from childhood to adulthood, but that this effect is lost in older age. This result is in conformance with that of Wilson and collaborators [50] who found that this capacity to enhance performance during the consolidation period is lost in older age, for this specific task. Previous research has already demonstrated that the brain suffers huge plasticity phenomena each time a new memory is learned [for review see 54]. However, this plasticity is further subject to changes with aging, as certain brain areas seem to be more vulnerable to the aging process [for review see 55].

The hippocampus is one of the brain structures that changes its functionality, decreasing its efficiency with age [27]. Considering the hippocampus to be an important structure that, together with the striatum is implicated in the consolidation process [25, 56], it might be possible to re-adapt the training and rehabilitation plans in Seniors, by means of alternative tasks requiring mostly different brain structures in order to optimize their performance gains.

In agreement with Dorfberger and collaborators [57], we also found sequence specific post-training gains in performance in Children performing a motor sequence (Finger Tapping Sequence task). Their data did not reveal any specific differences between Children ( 9 and 12 year olds) and Adolescents (17 year olds). Our results share the same trend also in older Age groups - Young and Mature Adults. Here, however, we show that Seniors lose this post-training advantage. Crucially, we also show that initial (T0) and post-training performances (T1 and T2) are superior in Young Adults and regress again in the older Age groups. Importantly a male advantage arises across 
testing points in the Young Adults. This male advantage is lost in Mature Adults, whereby the only advantage occurs in females in T2 - the offline post training gains. The female advantage in performance is maintained in T2 in Seniors and also reappears in T1 in this Age-group. As it seems, age and sex seem to concur to bias the complex motor processing and motor memory formation mechanisms. The development of such biases is not present in childhood, but soon appears in Young Adults and inverts its direction as adults mature. It seems, however, that this sinuous development is task-specific. Specifically, in the Go/No-Go task, differences in performance between sexes arise only in Mature Adults and are maintained stable in Seniors, whereby females outperform males in both T1 and T2.

Previous studies have shown that motor performance is influenced by age and sex differences from early childhood [58]. It has been well established that these early gender differences are task specific [59]. Although we did not find such differences so early on, as they are possibly more tenuous in early motor skill development, we did find these consolidated differences in the Finger Tapping Sequence task in Young Adults.

Moreno-Briseño and collaborators [60] have recently suggested that different learning mechanisms, like strategic calibration and spatial alignment, may contribute differently according to gender. This might explain different sex biases according to task. But how can we explain different biases alternating with age in the same task? Other studies have shown that skill proficiency in childhood is predictive of future skill proficiency [61]. However, and according to our own results, the matter seems quite more complex. A possible explanation might stem from Weiermann and Meier's [62] recent work, whereby they show that different learning processes are implicated in learning a specific sequence, depending on age. In particular, the authors show that performance of children and older adults highly depends on the existence of explicit knowledge, i.e., the presence of the training sequence. However, young adults (aged 20 to 30 years) do not reduce performance, independently of the presence of such explicit cues. It is possible that the activation of different neural networks, associated to the different processing strategies be involved across Age groups [e.g., 29, 63]. Specifically, explicit knowledge learningdependent performance might be attributed to a lower striatal function that is sometimes compensated by the activation of other areas, such as the frontal cortex [62].
As stated before, age-related changes in connectivity and preservation of cognitive functions in older adults has been associated to compensatory mechanisms associated with neuroanatomical and functional changes that lead to an overall increase and less efficient, functional connectivity [32]. Our results show that aging may alter the connectivity of brain networks underlying motor learning by increasing the bilateral-frontal and fronto-parietal connectivity [33]. Thus, it is possible that the changes in connectivity compromise the enhancement without additional training that is expected during the consolidation period and observed at younger ages. Accordingly, age can be a limiting factor in terms of performance gains without additional training. Despite the group's lower performance, the senior group was able to learn a new motor sequence. Further research is needed, however, in order to fully understand the neural correlates and differences across different Age groups and how these differences might be reduced in older people.

Despite the simplicity of the Go/No-Go task, a decision making task tapping executive functions (in particular inhibition response) there were no statistically significant improvements in performance, 24 hours post-training in any of the Age groups. Overnight sleep has been identified as essential for the activation of areas that are implicated in faster and more precise mapping of key-presses [64], however, we did not find such improvement in performance driven by motormemory plasticity. It seems clear that the processes implicated in motor memory formation are distinct from those implicated in response inhibition. Verbruggen and Logan [51], for example, have suggested that learning a stimulus-stop association through training would create an inhibitory tag that would be retrieved in future phases. Our results do not counter this suggestion, as performance in T2 was no different from performance in T1 in any of the groups. Indeed, in a study with preschool children, Thorell and collaborators [65] have shown that despite improvements in working memory with training, no such improvements were observed in inhibition. The authors go on to suggest that this might be due to the psychological and neural processes underpinning these distinct executive functions.

Although there were no differences in Consolidation, we did find sex differences in performance after practice (T1), given by better performances of Mature Adult and Senior Females. An animal study investigating learning and inhibition [66] has shown that despite the absence of significant 
differences in baseline activity, males and females differ in their ability to form conditioned associations and inhibit responses after practice trials. It is possible that the same applies to humans. Moreover, it has been suggested that gender differences in inhibitory control, might be related to the different inhibitory demands pressing on each gender during evolution [67]. This thesis has been recently supported by Hosseini-Kamkar and Morton [68], who put forth an evolutionary perspective to explain a female advantage in inhibitory control. However, the authors suggest that a less impulsive behaviour in females is not a trait, but a strategy employed during potentially reproductive periods. This is quite surprising, as we did not observe a female advantage in the Go/No-Go task in Young Adults, but in Mature Adults and Seniors instead. This incongruency might be explained by a recent study by Thakkar and collaborators [69] who did not find any sex differences in accuracy or response inhibition in a stopsignal task, but women did show greater sensitivity to trial history (flexible adjustments in speed-accuracy trade-offs and greater cognitive flexibility associated with response control). This could account for the improvement in performance in T1 (after practice trials) given by females. It is possible that no such difference between female and male participants was found in Young Adults as they might have already been performing at ceiling level in $\mathrm{TO}$.

As it seems, Age, Sex and Task all influence performance that is differently modulated as a result of these interacting factors. More research is needed in order to understand the distinct neural underpinnings of men and women and how it might be possible to enhance the individual gender capacity to learn and consolidate new motor memories, while delaying agedependent impairments by potentiating neural plasticity.

\section{REFERENCES}

[1] Mang CS, Campbell KL, Ross CJ, Boyd LA. Promoting neuroplasticity for motor rehabilitation after stroke: Considering the effects of aerobic exercise and genetic variation on brain-derived neurotrophic factor. Phys Ther 2013; 93: 1707-1716.

[2] Robertson EM, Pascual-Leone A, Miall RC. Current concepts in procedural consolidation. Nat Rev Neurosci 2004; 5: 576582.

[3] Kantak SS, Sullivan KJ, Fisher BE, Knowlton BJ, Winstein CJ. Neural substrates of motor memory consolidation depend on practice structure. Nat Neurosci 2010; 13: 923925.

[4] Pereira T, Abreu AM, Castro-Caldas A. Understanding task and expertise-specific motor acquisition and motor memory formation and consolidation. Percept Mot Skills 2013; 117: 108-129.
[5]

Ofen N, Shing YL. From perception to memory: Changes in memory systems across the lifespan. Neurosci Biobehav Rev 2013; 37: 2258-2267.

[6] Cai L, Chan JS, Yan JH, Peng K. Brain plasticity and motor practice in cognitive aging. Front Aging Neurosci 2014; 6: 31.

[7] Rebok GW, Ball K, Guey LT, Jones RN, Kim HY, King JW, et al. Ten-year effects of the advanced cognitive training for independent and vital elderly cognitive training trial on cognition and everyday functioning in older adults. J Am Geriatr Soc 2014; 62: 16-24.

[8] Taubert M, Draganski B, Anwander A, Muller K, Horstmann $A$, Villringer $A$, et al. Dynamic properties of human brain structure: Learning-related changes in cortical areas and associated fiber connections. J Neurosci 2010; 30: 1167011677.

[9] Engvig A, Fjell AM, Westlye LT, Moberget T, Sundseth $\varnothing$, Larsen VA, et al. Memory training impacts short-term changes in aging white matter: A longitudinal diffusion tensor imaging study. Hum Brain Mapp 2012; 33: 2390-2406.

[10] Nemeth D, Janacsek K, Fiser J. Age-dependent and coordinated shift in performance between implicit and explicit skill learning. Front Comput Neurosci 2013; 7: 147.

[11] Volkow ND, Logan J, Fowler JS, Wang GJ, Gur RC, Wong C, et al. Association between age-related decline in brain dopamine activity and impairment in frontal and cingulate metabolism. Am J Psychiatry 2000; 157: 75-80.

[12] Sowell ER, Peterson BS, Thompson PM, Welcome SE, Henkenius AL, Toga AW. Mapping cortical change across the human life span. Nat Neurosci 2003; 6: 309-315.

[13] Salat DH, Buckner RL, Snyder AZ, Greve DN, Desikan RS, Busa $\mathrm{E}$, et al. Thinning of the cerebral cortex in aging. Cereb Cortex 2004; 14: 721-730.

[14] Fotenos AF, Snyder AZ, Girton LE, Morris JC, Buckner RL. Normative estimates of cross-sectional and longitudinal brain volume decline in aging and AD. Neurology 2005; 64: 10321039.

[15] Sullivan EV, Pfefferbaum A. Diffusion tensor imaging and aging. Neurosci Biobehav Rev 2006; 30: 749-761.

[16] Rolland Y, Abellan van Kan G, Vellas B. Healthy brain aging: Role of exercise and physical activity. Clin Geriatr Med 2010; 26: 75-87.

[17] Voss MW, Prakash RS, Erickson KI, Basak C, Chaddock L, $\mathrm{Kim}$ JS, et al. Plasticity of brain networks in a randomized intervention trial of exercise training in older adults. Front Aging Neurosci 2010; 2: 32.

[18] Voss MW, Erickson KI, Prakash RS, Chaddock L, Kim JS, Alves $\mathrm{H}$, et al. Neurobiological markers of exercise-related brain plasticity in older adults. Brain Behav Immun 2013; 28: 90-99.

[19] Erickson KI, Weinstein AM, Lopez OL. Physical activity, brain plasticity, and Alzheimer's disease. Arch Med Res 2012; 43: 615-621.

[20] Berchicci M, Lucci G, Di Russo F. Benefits of physical exercise on the aging brain: The role of the prefrontal cortex. J Gerontol A Biol Sci Med Sci 2013; 68: 1337-1341.

[21] Galea JM, Vazquez A, Pasricha N, de Xivry JJ, Celnik P. Dissociating the roles of the cerebellum and motor cortex during adaptive learning: The motor cortex retains what the cerebellum learns. Cereb Cortex 2011; 21: 1761-1770.

[22] Orban $P$, Peigneux $P$, Lungu $O$, Albouy $G$, Breton E, Laberenne $F$, et al. The multifaceted nature of the relationship between performance and brain activity in motor sequence learning. Neuroimage, 2010; 49: 694-702.

[23] Steele CJ, Penhune VB. Specific increases within global decreases: A functional magnetic resonance imaging investigation of five days of motor sequence learning. $\mathrm{J}$ Neurosci 2010; 30: 8332-8341. 
[24] Landi SM, Baguear F, Della-Maggiore V. One week of motor adaptation induces structural changes in primary motor cortex that predict long-term memory one year later. $\mathrm{J}$ Neurosci 2011; 31: 11808-11813.

[25] Albouy G, Sterpenich V, Balteau E, Vandewalle G, Desseilles M, Dang-Vu T, et al. Both the hippocampus and striatum are involved in consolidation of motor sequence memory. Neuron 2008; 58: 261-272.

[26] Gheysen F, Van Opstal F, Roggeman C, Van Waelvelde H, Fias W. Hippocampal contribution to early and later stages of implicit motor sequence learning. Exp Brain Res 2010; 202: 795-807.

[27] Miller DB, O'Callaghan JP. Effects of aging and stress on hippocampal structure and function. Metabolism 2003; 52: 17-21.

[28] Rieckmann A, Bäckman L. Implicit learning in aging: Extant patterns and new directions. Neuropsychol Rev 2009; 19: 490-503.

[29] Rieckmann A, Fischer H, Bäckman L. Activation in striatum and medial temporal lobe during sequence learning in younger and older adults: Relations to performance. Neuroimage 2010; 50: 1303-1312.

[30] Robertson Z. Lifelong learning and the aging brain. In: Bell SC, editor. Senior law handbook. Colorado: Continuing Legal Education in Colorado, Inc; 2014. p. 405-411.

[31] Hampson M, Tokoglu F, Shen X, Scheinost D, Papademetris $\mathrm{X}$, Constable RT. Intrinsic brain connectivity related to age in young and middle aged adults. PLoS One 2012; 7: e44067.

[32] Meunier D, Stamatakis EA, Tyler LK. Age-related functional reorganization, structural changes, and preserved cognition. Neurobiol Aging 2014; 35: 42-54.

[33] Lin $\mathrm{CH}$, Chiang $M C, \mathrm{Wu} A D$, lacoboni $M$, Udompholkul $P$, Yazdanshenas $\mathrm{O}$, et al. Enhanced motor learning in older adults is accompanied by increased bilateral frontal and frontoparietal connectivity. Brain Connect 2012; 2: 56-68.

[34] Degardin A, Devos D, Cassim F, Bourriez JL, Defebvre L, Derambure $\mathrm{P}$, et al. Deficit of sensorimotor integration in normal aging. Neurosci Lett 2011; 498: 208-212.

[35] Zimerman M, Nitsch M, Giraux P, Gerloff C, Cohen LG, Hummel FC. Neuroenhancement of the aging brain: Restoring skill acquisition in old subjects. Ann Neurol 2012; 73: 10-15.

[36] Smith CD, Walton A, Loveland AD, Umberger GH, Kryscio RJ, Gash DM. Memories that last in old age: motor skill learning and memory preservation. Neurobiol Aging 2005; 26: 883-90

[37] Dorfberger S, Adi-Japha E, Karni A. Reduced susceptibility to interference in the consolidation of motor memory before adolescence. PLoS One 2007; 2: e240.

[38] Dorfberger S, Adi-Japha E, Karni A. Sex differences in motor performance and motor learning in children and adolescents: An increasing male advantage in motor learning and consolidation phase gains. Behav Brain Res 2009; 198: 165171.

[39] Krakauer JW, Shadmehr R. Consolidation of motor memory. Trends Neurosci 2006; 29: 58-64.

[40] Walker MP, Brakefield T, Morgan A, Hobson JA, Stickgold R. Practice with sleep makes perfect: Sleep dependent motor skill learning. Neuron 2002; 35: 205-211.

[41] Walker MP, Brakefield T, Hobson JA, Stickgold R. Dissociable stages of human memory consolidation and reconsolidation. Nature 2003; 425: 616-620.

[42] Kuriyama K, Stickgold R, Walker MP. Sleep-dependent learning and motor-skill complexity. Learn Mem 2004; 11: 705-713.

[43] Michel E. Motor coordination and executive functions. Dev Med Child Neurol 2012; 54: 971.
[44] Bruyer R, Brysbaert M. Combining speed and accuracy in cognitive psychology: Is the inverse efficiency score (IES) a better dependent variable than the mean reaction time (RT) and the percentage of errors (PE)? Psychol Belg 2011; 51: 513.

[45] Rach S, Diederich A, Colonius $H$. On quantifying multisensory interaction effects in reaction time and detection rate. Psychol Res 2010; 75: 77-94.

[46] Townsend JT, Ashby FG. Stochastic modeling of elementary psychological processes. Cambridge: Cambridge University Press; 1983.

[47] Fitts PM. The information capacity of the human motor system in controlling the amplitude of movement. J Exp Psychol Gen 1992; 121: 262-269.

[48] Nemeth D, Janacsek K. The dynamics of implicit skill consolidation in young and elderly adults. J Gerontol B Psychol Sci Soc Sci 2011; 66: 15-22.

[49] Fogel SM, Albouy G, Vien C, King BR, Hoge RD, Jbabdi S, et al. Age-related differences in cerebral activation for motor sequence learning are correlated with sleep spindles. J. Sleep Res 2012; 21: 5.

[50] Wilson JK, Baran B, Pace-Schott EF, Ivry RB, Spencer RMC Sleep modulates word-pair learning but not motor sequence learning in healthy older adults. Neurobio Aging 2012; 33: 991-1000

[51] Verbruggen F, Logan GD. Automatic and controlled response inhibition: Associative learning in the go/no-go and stop-signal paradigms. J Exp Psychol Gen 2008; 137: 649672.

[52] Garavan H, Ross TJ, Murphy K, Roche RAP, Stein, EA Dissociable executive functions in the dynamic control of behavior: inhibition, error detection, and correction. Neuroimage 2002; 17: 1820-1829.

[53] Leversen JSR, Haga M, Sigmundsson $\mathrm{H}$. From children to adults: Motor performance across the life-span. PLoS One 2012; 7: e38830.

[54] Dayan E, Cohen LG. Neuroplasticity subserving motor skill learning. Neuron 2011; 72: 443-454.

[55] Burke SN, Barnes CA. Neural plasticity in the ageing brain Nat Rev Neurosci 2006; 7: 30-40.

[56] Albouy G, Sterpenich V, Vandewalle G, Darsaud A, Gais S Rauchs $\mathrm{G}$, et al. Interaction between hippocampal and striatal memory systems predicts subsequent motor sequence memory consolidation. PLoS One 2013; 8: e59490.

[57] Dorfberger S, Adi-Japha E, Karni A. Sequence specific motor performance gains after memory consolidation in children and adolescents. PloS One 2012; 7: e28673.

[58] Toriola AL, Igbokwe NU. Age and sex differences in motor performance of pre-school Nigerian children. J Sports Sci 1986; 4: 219-227.

[59] Raudsepp L, Paasuke M. Gender differences in fundamental movement patterns, motor performances, and strength measurements of prepubertal children. Pediatr Exerc Sci 1995; 7: 294-304

[60] Moreno-Briseño P, Díaz R, Campos-Romo A, FernandezRuiz J. Sex-related differences in motor learning and performance. Behav Brain Funct 2010; 6: 74.

[61] Barnett LM, van Beurden E, Morgan PJ, Brooks LO, Beard $J R$. Gender differences in motor skill proficiency from childhood to adolescence. Res Q Exerc Sport 2010; 81: 162170.

[62] Weiermann B, Meier B. Incidental sequence learning across the lifespan. Cognition 2012; 123: 380-391.

[63] Dennis NA, Cabeza R. Age-related dedifferentiation of learning systems: An fMRI study of implicit and explicit learning. Neurobiol Aging 2011; 32: 2318.e17. 
[64] Walker MP, Stickgold R, Alsop D, Gaab N, Schlaug G. Sleep-dependent motor memory plasticity in the human brain. Neuroscience 2005; 133: 911-917.

[65] Thorell LB, Lindqvist S, Bergman NS, Bohlin G, Klingberg T. Training and transfer effects of executive functions in preschool children. Dev Sci 2009; 12: 106-113.

[66] Bucci DJ, Hopkins ME, Keene CS, Sharma M, Orr LE. Sex differences in learning and inhibition in spontaneously hypertensive rats. Behav Brain Res 2008; 187: 27-32.
[67] Yuan J, He Y, Qinglin Z, Chen A, Li H. Gender differences in behavioral inhibitory control: ERP evidence from a twochoice oddball task. Psychophysiology 2008; 45: 986-993.

[68] Hosseini-Kamkar N, Morton JB. Sex differences in selfregulation: An evolutionary perspective. Front Neurosci 2014; 8: 233.

[69] Thakkar KN, Congdon E, Poldrack, RA, Sabb FW, London $\mathrm{ED}$, Cannon TD, et al. Women are more sensitive than men to prior trial events on the Stop-signal task. $\mathrm{Br} \mathrm{J}$ Psychol 2014; 105: 254-272.

(C) 2014 Pereira et al.; Licensee Cosmos Scholars Publishing House.

This is an open access article licensed under the terms of the Creative Commons Attribution Non-Commercial License

(http://creativecommons.org/licenses/by-nc/3.0/), which permits unrestricted, non-commercial use, distribution and reproduction in any medium, provided the work is properly cited. 